\title{
Lymphedema-distichiasis syndrome
}

\author{
Sean McDermott MB, Conor Lahiff MD
}

Competing interests: None declared.

This article has been peer reviewed.

The authors have obtained patient consent.

Affiliation: St. James's Hospital and Trinity College, Dublin, Ireland

Correspondence to: Conor Lahiff, conorlahiff @physicians.ie

CMAJ 2016. DOI:10.1503 /cmaj.141029
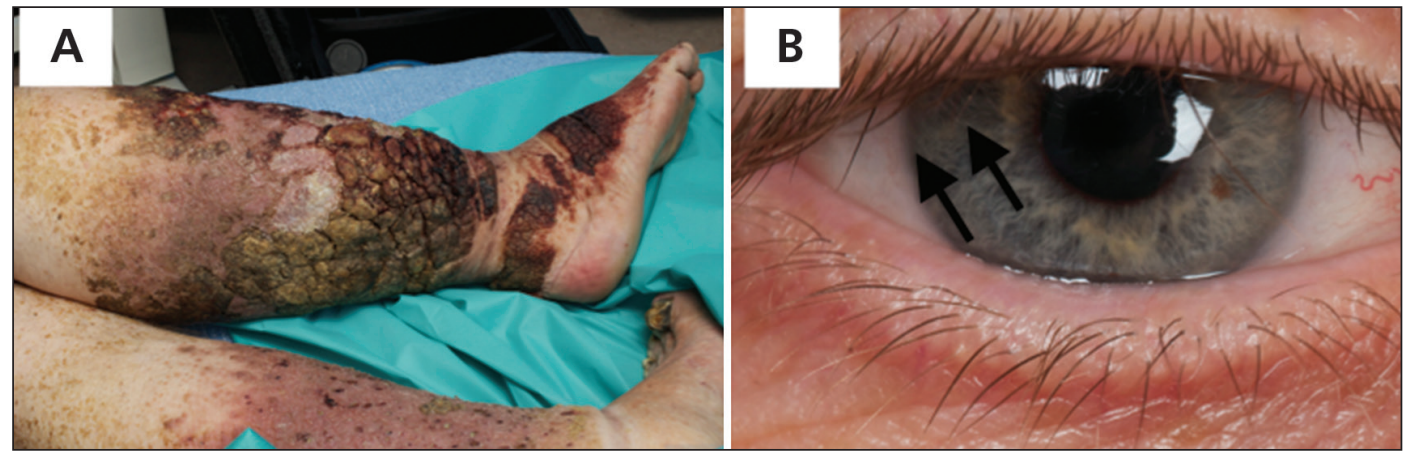

Figure 1: (A) Bilateral lymphedema in the legs associated with hyperkeratotic plaques and fissuring to midshin level. (B) Patient's right eye showing a second layer of eyelashes arising from the meibomian glands and directed toward the cornea (arrows).

$\mathrm{A}$ 54-year-old man presented to the emergency department with bilateral swelling in his legs that was progressive, longstanding and had started when the patient was in his midthirties. Hyperkeratotic plaques and fissuring to midshin level on both legs also were observed (Figure 1A). He was otherwise healthy, with normal levels for liver enzymes and serum creatinine, and a negative result for protein on 24-hour urinalysis. Distichiasis (i.e., abnormal growth of eyelashes from the meibomian glands) was visible on closer physical examination of his eyelids (Figure 1B). Lymphedema-distichiasis syndrome was diagnosed, and his legs responded well to bathing and débridement.

The patient's two brothers, grandmother and a maternal uncle also had lymphedema. Both brothers were in their late twenties when the condition developed, but no information was available for his uncle and grandmother. There was no reported family history of distichiasis, and genetic testing was unavailable.

Most cases of lymphedema are secondary and have infectious or iatrogenic causes. Primary

Clinical images are chosen because they are particularly intriguing, classic or dramatic. Submissions of clear, appropriately labelled high-resolution images must be accompanied by a figure caption and the patient's written consent for publication. A brief explanation (250 words maximum) of the educational significance of the images with minimal references is required. lymphedema occurs in 1-3 out of every 10000 live births, ${ }^{1}$ with a differential diagnosis that includes Milroy disease, lymphedema-distichiasis syndrome and hypotrichosis-lymphedema-telangiectasia syndrome. Primary lymphedema can also be a feature of certain inherited chromosomal conditions, such as Aagenaes syndrome, Noonan syndrome, Klinefelter syndrome, Turner syndrome, and trisomy 13 , 18,21 and $22 .{ }^{2}$

Lymphedema-distichiasis syndrome is a congenital lymphedema associated with the presence of aberrant eyelashes arising from the meibomian glands. ${ }^{3,4}$ It is a clinical diagnosis and can be associated with mutations in the FOXC2 gene; ${ }^{3}$ inheritance is autosomal dominant with variable penetrance. ${ }^{4}$ Bilateral lymphedema of the legs is usually present in the patient by age 30 years. Distichiasis is usually present at birth and can cause corneal irritation or recurrent conjunctivitis ${ }^{5}$ in up to $75 \%$ of patients; however, it was asymptomatic in this patient.

\section{References}

1. Kurland LT, Molgaard CA. The patient record in epidemiology. Sci Am 1981;245:54-63.

2. Saito Y, Nakagami H, Kaneda Y, et al. Lymphedema and therapeutic lymphangiogenesis. BioMed Res Int 2013:804675.

3. Connell F, Brice G, Mortimer P. Phenotypic characterization of primary lymphedema. Ann N Y Acad Sci 2008;1131:140-6.

4. Johnson SM, Kincannon JM, Horn TD. Lymphedema-distichiasis syndrome: report of a case and review. Arch Dermatol 1999; 135:347-8.

5. Allen RC. Genetic diseases affecting the eyelids: What should a clinician know? Curr Opin Ophthalmol 2013;24:463-77. 